\title{
Regulation of $\delta$ Opioid Receptor-Mediated Signaling and Antinociception in Peripheral Sensory Neurons by Arachidonic Acid-Dependent 12/15-Lipoxygenase Metabolites $\$$
}

\author{
Laura C. Sullivan, Teresa A. Chavera, Xiaoli Gao, Miryam M. Pando, and Kelly A. Berg \\ Department of Pharmacology (L.C.S., T.A.C., M.P., K.A.B.) and Institutional Mass Spectrometry Laboratory (X.G.). University of \\ Texas Health Science Center, San Antonio, Texas
}

Received April 23, 2017; accepted April 27, 2017

\begin{abstract}
The function of $\delta$ opioid receptors (DOR) expressed by peripheral painsensing neurons (nociceptors) is regulated by both cyclooxygenaseand lipoxygenase (LOX)-dependent arachidonic acid (AA) metabolites. Whereas cyclooxygenase metabolites enhance responsiveness, LOX metabolites elicit a refractory, nonsignaling state of the DOR receptor system for antinociceptive signaling. In this study, using high-performance liquid chromatography-tandem mass spectrometry analyses, we have found that the 12-/15-LOX metabolites, 12-hydroxyeicosatetraenoic acid (HETE) and 15-HETE, were elevated after treatment of adult rat primary sensory neuron cultures with AA. Exogenously applied 12-HETE and 15-HETE, but not 5 -HETE, completely prevented DOR and $\kappa$ opioid receptor (KOR) agonist-mediated inhibition of prostaglandin E2 $\left(\mathrm{PGE}_{2}\right)$-stimulated cAMP accumulation, but not inhibition, by the $5-\mathrm{HT}_{1}$ receptor
\end{abstract}

agonist 5-carboxamidotryptamine in cultured peripheral sensory neurons and in Chinese hamster ovary $(\mathrm{CHO})$ cells heterologously expressing DOR or KOR. Similarly, intraplantar injection of 12- or 15-HETE, either alone or in combination, prevented DOR agonistmediated inhibition of $\mathrm{PGE}_{2}$-evoked thermal allodynia. Further, both AA- and carrageenan-mediated induction of the nonresponsive state of the DOR system was blocked by an intraplantar coinjection of the 12-/15-LOX inhibitors baicalein and luteolin. In contrast to the regulation of CAMP signaling, pretreatment with 12- and 15-HETE had no effect on either DOR or KOR agonist- mediated activation of extracellular signal-regulated kinase in peripheral sensory neurons or $\mathrm{CHO}$ cells. These results suggest that the analgesic efficacy of peripherally restricted opioids for treatment of inflammatory pain may be enhanced by adjunct inhibition of LOX activity.

\section{Introduction}

Treatment of pain disorders continues to be a major clinical challenge. Although drugs that target the $\mu$ opioid receptor (MOR; e.g., morphine and its analogs) are used most often for the treatment of moderate to severe pain, significant central nervous system (CNS)-mediated adverse effects limit their use. As an alternative to MOR, the $\delta$ opioid receptor (DOR) has attracted interest as a possible target for analgesic drug development (for review, see Gendron et al., 2015).

In addition to neurons in the CNS, opioid receptors [MOR, DOR, and $\kappa$ opioid receptors (KOR)] are expressed on peripheral afferent pain-sensing neurons (nociceptors). Although peripheral MOR, DOR, and KOR are functionally inactive for analgesia under most basal conditions, they can produce profound analgesia when tissue is damaged or inflammation is present in the peripheral tissue (Stein and Lang, 2009; Stein, 2016). Similarly, in a rat behavioral model of nociception, peripheral opioid receptors are nonresponsive to agonist

https://doi.org/10.1124/jpet.117.241604.

S This article has supplemental material available at jpet.aspetjournals.org. for antinociception, but after a brief pretreatment with an inflammatory mediator, such as bradykinin (BK) or arachidonic acid (AA), they become functionally competent to reduce thermal allodynia (Rowan et al., 2009; Berg et al., 2011; Sullivan et al., 2015). Moreover, in an ex vivo model system (i.e., primary cultures of peripheral sensory neurons), opioid receptor agonists do not inhibit adenylyl cyclase activity or neuropeptide release; however, brief pretreatment ("priming") with BK or AA induces opioid receptor system functional competence for antinociceptive signaling (Patwardhan et al., 2005; Patwardhan et al., 2006; Berg et al., 2007a,b; Sullivan et al., 2015). This induction of responsiveness of peripheral opioid receptor antinociceptive systems is mediated by a cyclooxygenase-dependent metabolite of $\mathrm{AA}$ and is transient, returning to a nonresponsive state within 30-60 minutes (Berg et al., 2007a, 2011; Sullivan et al., 2015).

Recently, we discovered that DOR responsiveness is also regulated by a lipoxygenase (LOX)-sensitive metabolite of AA in peripheral sensory neurons (Sullivan et al., 2015). After induction of functional competence by administration of exogenous AA or carrageenan to the hind paw, the DOR system subsequently becomes unresponsive to agonist-stimulated

ABBREVIATIONS: AA, arachidonic acid; ALA, $\alpha$-linolenic acid; BAIC, baicalein; BK, bradykinin; CHO, Chinese hamster ovary; CNS, central nervous system; DOR, $\delta$ opioid receptor; DPDPE, [D-Pen ${ }^{2,5}$ ]-enkephalin; ERK, extracellular signal-regulated kinase; 5-HETE, 5hydroxyeicosatetraenoic; 12-HETE, 12- hydroxyeicosatetraenoic; 15-HETE, 15- hydroxyeicosatetraenoic; HBSS, Hanks' balanced salt solution; HPLC, high-performance liquid chromatography; LA, linolenic acid; LC-MS/MS, liquid chromatography-tandem mass spectrometry; LOX, lipoxygensase; LTA4, leukotriene A4; LUT, luteolin; pERK, phosphorylated ERK; PGE2, prostaglandin $E_{2}$; PWL, paw withdrawal latency. 
antinociceptive signaling. This unresponsive state differs from that of the basal condition in that it is refractory to reinduction of functional competence by BK; however, treatment with LOX inhibitors permits reinduction of DOR functional competence by $\mathrm{BK}$, indicating that the unresponsive state of the DOR system that follows AA or carrageenan is produced by LOX-dependent AA metabolites.

In this study, using high-performance liquid chromatography (HPLC) tandem mass spectrometry analyses (LC/MS/MS), we have identified LOX-dependent AA metabolites that are involved in producing the refractory, nonresponsive state of the DOR system for antinociceptive signaling in adult rat ex vivo and in vivo model systems. Results of this study suggest that the duration of peripheral opioid receptor responsiveness under inflammatory conditions may be increased by inhibition of 12-/15-LOX and further underscore the unique regulation of the function of opioid receptors expressed by peripheral sensory neurons.

\section{Materials and Methods}

Materials. Bradykinin (BK), [D-Pen $\left.{ }^{2,5}\right]$-enkephalin (DPDPE), U50488, 5-carboxamidotryptamine (5-CT), rolipram, and $\lambda$-carrageenan were purchased from Sigma-Aldrich (St Louis, MO); prostaglandin $\mathrm{E}_{2}$ $\left(\mathrm{PGE}_{2}\right)$, 5-hydroxyeicosatetraenoic (HETE), 12-HETE, 15-HETE, luteolin (LUT), baicalein (BAIC), AA, and the LC-MS lipid standards (12-HETE, 15-HETE, and 5-HETE) were purchased from Cayman Chemicals (Ann Arbor, MI); The LC-MS lipid standards, eicosapentaenoic acid (EPA), leukotriene A4 (LTA4), linolenic acid (LA), and $\alpha$-linolenic acid (ALA) were purchased from Avanti Polar Lipids (Alabaster, AL); $\left[{ }^{125} \mathrm{I}\right]-\mathrm{cAMP}$ was purchased from PerkinElmer Life and Analytical Sciences (Boston, MA). For tissue cultures, collagenase was purchased from Worthington (Lakewood, NJ), and all other culture reagents were purchased from Invitrogen Corp (Carlsbad, CA). HPLCgrade acetonitrile and isopropanol were purchased from Fisher Scientific (Pittsburg, PA); MS-grade ammonium acetate was purchased from Fluka (St. Louis, MO).

Animals. Adult male Sprague-Dawley rats (Charles River, Wilmington, MA) weighing 250-300 g were used for all behavioral and cell culture studies. Animals were housed for at least 1 week, with food and water available ad libitum before behavioral testing or harvesting of trigeminal ganglion cells. Animal protocols were approved by the Institutional Animal Care and Use Committee of the University of Texas Health Science Center at San Antonio and conformed to the International Association for the Study of Pain and federal guidelines.

Primary Culture of Rat Peripheral Sensory Neurons. Primary cultures of adult male rat trigeminal ganglion cells were prepared as previously described (Berg et al., 2007a, 2011, 2012). Briefly, fresh trigeminal ganglia were washed with Hanks' balanced salt solution (HBSS; $\mathrm{Ca}^{++}{ }_{-}, \mathrm{Mg}^{++}$-free), digested with $3 \mathrm{mg} / \mathrm{ml}$ collagenase (30 minutes at $37^{\circ} \mathrm{C}$ ), and centrifuged (1000 rpm, 1 minute). In the same solution, pellets were further digested with $0.1 \%$ trypsin (15 minutes) and $167 \mu \mathrm{g} / \mathrm{ml}$ DNase $\left(10\right.$ minutes at $\left.37^{\circ} \mathrm{C}\right)$. Next, cells were again centrifuged (2000 rpm, 2 minutes) and resuspended in high-glucose Dulbecco's modified Eagle's medium (DMEM) containing $100 \mathrm{ng} / \mathrm{ml}$ nerve growth factor, $10 \%$ fetal bovine serum, $1 \times$ Pen/Strep; $1 \times$ L-glutamine and mitotic inhibitors $(7.5 \mu \mathrm{g} / \mathrm{ml}$ uridine and $17.5 \mathrm{mg} / \mathrm{ml} \mathrm{5-fluoro-2}$-deoxyuridine). After trituration to disrupt tissue, cells were seeded on polylysine-coated 48-well (for signaling experiments) or 10-cm plates (for LC-MS/MS experiments). Media was changed 24 hours and 48 hours after plating. On the 5 th day of culture, cells were refed with serum-free DMEM without nerve growth factor. The cell-signaling assays and preparations of samples for mass spectrometry were performed on the 6th day of culture.

Chinese Hamster Ovary-K1 Cell Culture and Nucleofection. Chinese hamster ovary (CHO-K1 cells were maintained in minimal essential medium $\alpha$ formulation ( $\alpha$-MEM) supplemented with 5\% FBS; 24 hours before nucleofection, cells were seeded into T175 flasks (to provide $\sim 80 \%$ confluency). CHO cells were nucleofected with either mouse DOR or rat KOR cDNA cloned into pcDNA3 $\left(2 \times 10^{6}\right.$ cells, $100-\mu \mathrm{l}$ volume, $2 \mu \mathrm{g}$ of plasmid) using cell line kit $\mathrm{T}$ and nucleofector II according to the manufacturer's directions (Lonza AG, Basel, Switzerland). Nuclefected cells were seeded into 48 -well plates at a density of $10^{5}$ cells per well in $\alpha \mathrm{MEM}$ containing $10 \%$ FBS. Following a 24 hour plating period, cells were washed with HBSS) and placed into DMEM/F-12 [1:1] and cultured for an additional 24 hours before experiments.

Lipid Extraction. Lipids were extracted from samples prepared from primary cultures using cold $\left(-20^{\circ} \mathrm{C}\right)$ chloroform/methanol $(2: 1$, $\mathrm{v} / \mathrm{v}$ ) in a 5:1 ratio over the sample volume (Gao et al., 2012). The mixtures were vortexed for 10 seconds and allowed to stand on ice for 30 minutes, followed by vortexing for an additional 10 seconds. After centrifugation at $13,800 \mathrm{~g}$ for 10 minutes, $200 \mu \mathrm{l}$ of the chloroform layer was removed and vacuum dried. Before LC-MS analysis, the lipid extracts were reconstituted in $200 \mu \mathrm{l}$ of isopropanol containing $10 \mathrm{mM}$ ammonium acetate.

LC-MS/MS. Analysis of eicosanoids produced by treatment of primary cultures of peripheral sensory neurons was done as previously described (Gao et al., 2012). HPLC-ESI-MS/MS analyses were conducted on a Thermo Fisher Q Exactive fitted with a PicoChip nanospray source (New Objective, Woburn, MA) and a PicoChip column (Waters Atlantis dC18 column; $150 \mu \mathrm{m} \times 105 \mathrm{~mm} ; 3-\mu \mathrm{m}$ particles; Waters Corporation, Milford, MA). A 55-minute wateracetonitrile-isopropanol-ammonium acetate gradient was run at the flow rate of $1 \mu \mathrm{l} / \mathrm{min}$. Mobile phase A was acetonitrile/water (40:60) containing $10 \mathrm{mM}$ ammonium acetate, and mobile phase B was acetonitrile/isopropanol (10:90) containing $10 \mathrm{mM}$ ammonium acetate. LC gradient was from $10 \% \mathrm{~B}$ to $99 \% \mathrm{~B}$ over 33 minutes and held at $99 \% \mathrm{~B}$ for 15 minutes. Data-dependent analyses were conducted using one full MS scan $[\mathrm{m} / z 200-2000 ; 70,000$ resolution $(\mathrm{m} / z$ 300)] followed by six tandem MS scans with electrospray negative ion detection using normalized collision energy of 35 arbitrary units. Progenesis CoMet (Nonlinear Dynamics Limited, Durham, NC) was used to process the raw data files. Peak alignment and integration were performed, and the relative abundance was generated for each lipid among the different experimental groups. Fatty acids were identified by searching the following databases: METLIN (http:// metlin.scripps.edu/index.php); lipid maps (http://www.lipidmaps.org/ data/structure/); and HMDB (Human Metabolome Database; http:// www.hmdb.ca/) using a 5-ppm mass tolerance. The putative lipid identifications were manually verified through examination of the MS/MS and in comparison with the retention times from commercially available standards (Cayman Chemicals and Avanti Polar Lipids).

Measurement of Cellular cAMP Levels. Receptor-mediated inhibition of $\mathrm{PGE}_{2}$-stimulated adenylyl cyclase activity in cultured peripheral sensory neurons or CHO cells was measured as previously described (Sullivan et al., 2015). Briefly, cAMP accumulation was determined after a 15-minute incubation with the phosphodiesterase inhibitor rolipram $(0.1 \mathrm{mM})$, with or without the adenylyl cyclase activator, $\mathrm{PGE}_{2}(1 \mu \mathrm{M})$, alone or in combination with the DOR agonist (DPDPE, $100 \mathrm{nM}$ ), KOR agonist (U50488, $100 \mathrm{nM}$ ), or the $5-\mathrm{HT}_{1}$ receptor agonist (5-carboxamidotryptamine, 5-CT, $100 \mathrm{nM}$ ). Cells were washed twice with HBSS containing $20 \mathrm{mM}$ HEPES, pH 7.4 (buffer) followed by pretreatment with BK $(10 \mu \mathrm{M})$ or AA $(50 \mu \mathrm{M})$ for 15 minutes at $37^{\circ} \mathrm{C}$ in buffer. All reactions were terminated by aspiration of the buffer and addition of $500 \mu$ l ice-cold absolute ethanol. Ethanol extracts were dried and reconstituted in buffer (100 $\mu \mathrm{l} 50 \mathrm{mM}$ sodium acetate, $\mathrm{pH}$ 6.2), and the cAMP content was determined by radioimmunoassay.

To determine the effects of LOX metabolites on cAMP levels in primary cultures, cells were pretreated with either vehicle, 5-HETE (100 nM), 12-HETE (100 nM), 15-HETE (100 nM), or 12-HETE and 15-HETE combined, for 15-45 minutes followed by a 15-minute incubation with $\mathrm{BK}(10 \mu \mathrm{M})$ and subsequent measurement of basal 


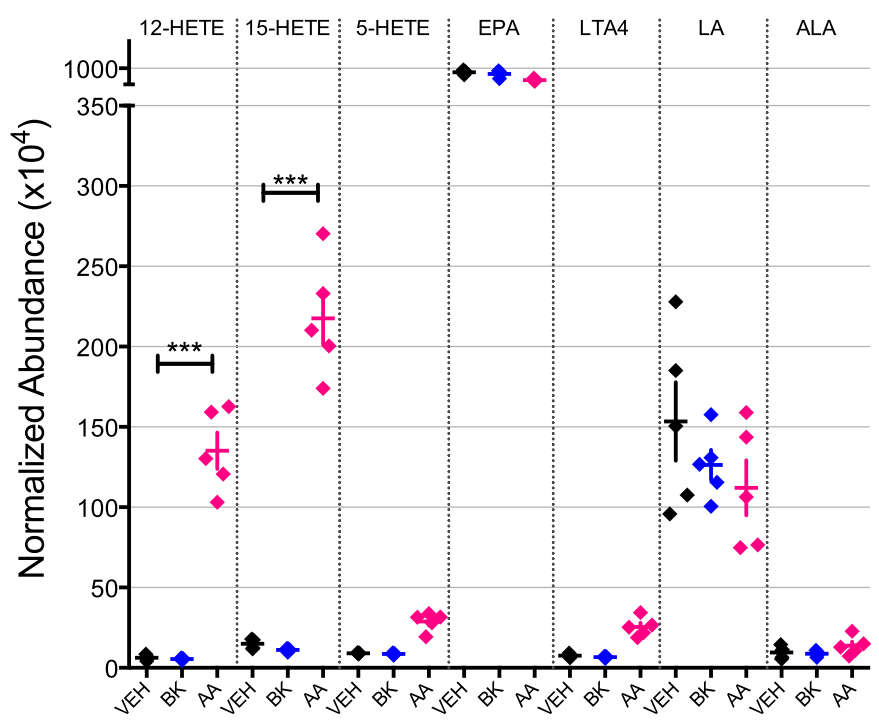

Fig. 1. Relative abundance of fatty acid metabolites in cultures of peripheral sensory neurons treated for 15 minutes with either vehicle (Veh), BK (10 $\mu \mathrm{M})$, or AA (50 $\mu \mathrm{M})$. Eicosanoids (12-HETE, 15-HETE, and 5-HETE), EPA, LTA4, LA, and ALA were identified by comparison with synthetic standards using LC-MS/MS, and the average accumulation of metabolites was calculated for each treatment. Levels of 12-HETE and 15-HETE were significantly greater in cultures treated with AA compared with Veh $(* * * P<0.001)$. Data represent mean \pm S.E.M. of five experiments.

and $\mathrm{PGE}_{2}$-stimuated cAMP levels, with and without receptor agonists. For experiments determining the effects of inhibition of 12- and 15-LOX for restoration of functional competence (Sullivan et al., 2015), we used a combination of the flavinoids, BAIC, and LUT, which have been shown to target selectively 12/15-LOX enzymes (Sadik et al., 2003; Deschamps et al., 2006; van Leyen et al., 2006; Lee and Kim, 2010). Briefly, cultures were washed and incubated with either vehicle or a combination of BAIC and LUT (each at 1- $\mu \mathrm{M}$ concentration) for 30 minutes before treatment with either AA or vehicle and an additional 15-minute incubation. Cells were washed and the responsiveness of DOR system was measured 15 minute or 60 minute later as described already.

To determine the effects 12- and 15-HETE on cAMP levels in CHO cells transiently expressing DOR or KOR, cells were pretreated with vehicle, 12-HETE (100 nM), 15-HETE (100 nM), or 12-HETE and 15-HETE combined for 15 minutes, followed by measurement of basal and $\mathrm{PGE}_{2}$-stimuated cAMP levels, with and without receptor agonists.

Measurement of Extracellular Signal-Regulated Kinase Activity. Receptor-mediated extracellular signal-regulated kinase (ERK) activation was determined by measuring phosphorylated ERK levels as described previously (Berg et al., 2011). Briefly, primary cultures or CHO cells transiently expressing either mouse DOR or rat KOR, washed twice with HBSS containing 20 mM HEPES, pH 7.4 (buffer), were preincubated for 15 minutes at $37^{\circ} \mathrm{C}$ (room air) with 12 and 15-HETE in combination (100 $\mathrm{nM}$ each) or vehicle. Cells were then further incubated with the DOR agonist DPDPE or the KOR agonist $\mathrm{U} 50488$ for $0-15$ minutes at $37^{\circ} \mathrm{C}$. When testing responses in primary sensory neuron cultures, $\mathrm{BK}(10 \mu \mathrm{M})$ was added during the 15 -minute preincubation period. Reactions were terminated by aspiration of buffer and addition of $50 \mu \mathrm{l}$ of lysis buffer supplied with the SureFire phospho-ERK (pERK) assay kit (PerkinElmer, Waltham, MA). Samples were processed according to the manufacturer's directions. The fluorescence signal from pERK was measured in duplicate using a Fluostar microplate reader (BMG Labtech, Offenburg, Germany) with AlphaScreen settings.

Measurement of Thermal Allodynia in Rat Hind Paws. Thermal allodynia was measured as previously described by observers blinded to treatment allocations (Rowan et al., 2009; Berg et al., 2011, 2012; Sullivan et al., 2015). Briefly, paw withdrawal latency (PWL) in response to a thermal (radiant heat) stimulus applied to the rat hind paw was measured before and after drug treatments (Hargreaves et al., 1988). The intensity of the radiant heat source was adjusted such that the average latency for paw withdrawal was $10 \pm 2$ seconds, with a cutoff of 25 seconds to avoid tissue damage. All drugs were

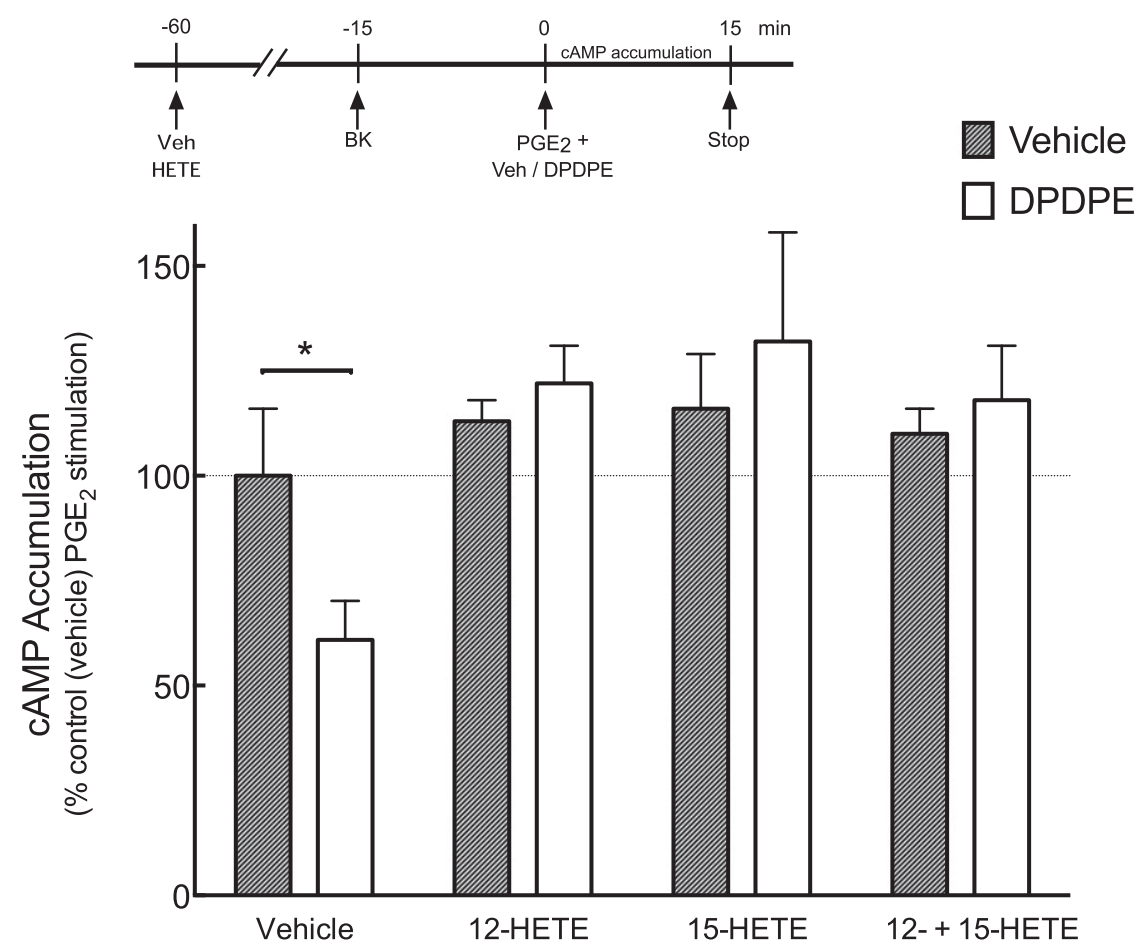

Fig. 2. Effects of the LOX-dependent AA metabolites, 12-HETE, and 15-HETE, either alone or in combination, on DPDPE-mediated inhibition of $\mathrm{PGE}_{2-}$ stimulated cAMP accumulation. Primary cultures of peripheral sensory neurons were pretreated with 12-HETE (100 nM), 15-HETE (100 nM), or 12-HETE and 15-HETE combined for 45 minutes before incubation with $\mathrm{BK}(10 \mu \mathrm{M})$ for an additional 15 minutes to induce DOR functional competence. The amount of cAMP accumulated $\left(15\right.$ minutes, $\left.37^{\circ} \mathrm{C}\right)$ in response to $\mathrm{PGE}_{2}(1 \mu \mathrm{M})$, with or without DPDPE (100 nM), was measured as described in Materials and Methods. Pretreatment with the HETEs had no effect on basal or $\mathrm{PGE}_{2}$-stimulated cAMP accumulation levels, which were $1.2 \pm 0.6 \mathrm{pmol} /$ well and $187 \% \pm 34 \%$ above basal, respectively. Data represent mean \pm S.E.M. of five experiments. ${ }^{*} P<0.05$ versus vehicle (no DPDPE).

\section{Treatment}


injected intraplantarly locally into the plantar surface of the hind paw in a volume of $50 \mu \mathrm{l}$. Drug effects were measured as the change (seconds) from baseline PWL over time. The PWL measurements were taken in duplicate at least 30 seconds apart, and the average was used for statistical analysis. In all experiments, rats received pretreatments (described later) followed by an injection of DPDPE $(20 \mu \mathrm{g})$ alone, $\mathrm{PGE}_{2}$ alone $(0.3 \mu \mathrm{g})$, or a coinjection of $\mathrm{PGE}_{2}(0.3 \mu \mathrm{g})$ and DPDPE $(20 \mu \mathrm{g})$. In experiments to test the effects of LOX metabolites, rats received intraplantarly injections of either vehicle, 12-HETE $(0.1 \mu \mathrm{g})$ or 15-HETE $(0.1 \mu \mathrm{g})$, alone or in combination, followed 45 minutes later with an injection of vehicle or BK $(25 \mu \mathrm{g})$ to produce functional competence. After BK injection, rats received intraplantar injections of vehicle $\mathrm{PGE}_{2}$ or a coinjection of $\mathrm{DPDPE}$ and $\mathrm{PGE}_{2}$. In the experiments using carrageenan to induce inflammation, rats received an initial injection of either vehicle or carrageen $(500 \mu \mathrm{g}, 50 \mu \mathrm{l}$, intraplantar). Allodynia was measured at 15 minutes and 3 hours after the carrageenan injection. To test the effect of 12/15-LOX inhibition for restoration of DOR functional competence, rats received carrageenan injections followed 2.5 hours later with an injection of either vehicle or a coinjection $(50 \mu \mathrm{l})$ of the $12 / 15$-LOX inhibitors, BAIC (3 $\mu \mathrm{g})$ and LUT (3 $\mu \mathrm{g}$ ) (Sadik et al., 2003; Deschamps et al., 2006), collectively referred to as LOX inhibitor in the figure legends. DOR functional competence was assessed 30 minutes after administration of the LOX inhibitors by intraplantar injection of DPDPE ( $20 \mu \mathrm{g})$. In all experiments, PWLs were measured every 5 minutes in duplicate over a 20-minute period after the final injection.

Data Analysis. Time-course data from behavioral and cellular experiments were analyzed with two-way analysis of variance followed by Tukey's post-hoc test to compare the treatment effects over time. Area under the time course curve for behavioral experiments was calculated for individual rats using the trapezoidal method with
Prism software (version 6.0, GraphPad Software, Inc., San Diego, CA) and is expressed as the mean \pm S.E.M. of each group. Statistical analyses of the area under the time course curve data were done with one-way analysis of variance followed by Dunnett's post hoc test to determine the significance from vehicle controls. For cellular experiments, statistical significance was assessed using one-way analysis of variance followed by Dunnett's post hoc test to compare group means of test agents to the corresponding vehicle pretreatment. For all experiments, data are presented as mean \pm S.E.M. and were analyzed using Prism software (version 6.0, GraphPad Software, Inc.). $P<0.05$ was considered statistically significant.

\section{Results}

Identification of LOX Metabolites Produced by Exogenous Application of AA. We have reported previously that treatment of peripheral sensory neurons in culture with AA for 15 minutes induces transient functional competence of the DOR system to inhibit adenylyl cyclase activity that is followed by a nonresponsive state that is resistant to reinduction of functional competence. This nonresponsive, refractory state does not occur after the induction of functional competence by treatment with $\mathrm{BK}$ and can be blocked by inhibitors of LOX (Sullivan et al., 2015). To identify the LOX-dependent metabolites of AA that lead to this refractory, nonresponsive state of the DOR system, we treated peripheral sensory neuron cultures for 15 minutes with vehicle, BK, or AA. As shown in Fig. 1, the eicosanoids 12-hydroxyeicosatetraenoic acid (12-HETE), 15-HETE, and

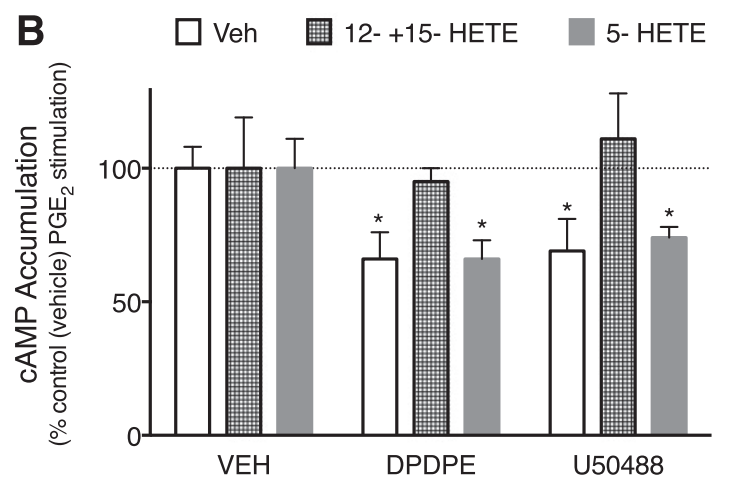

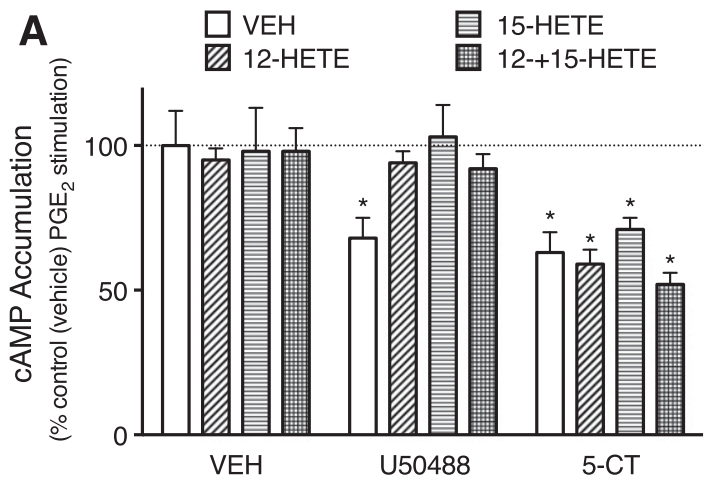

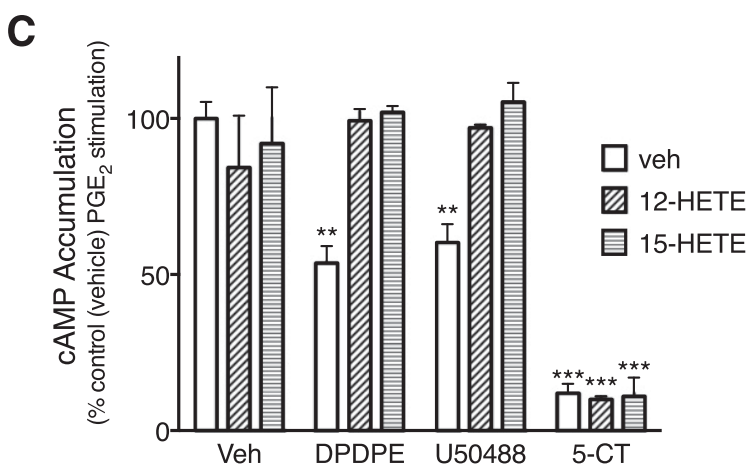

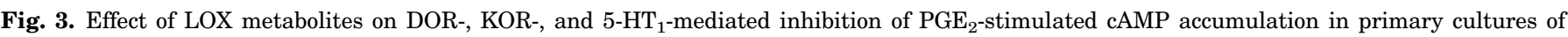
peripheral sensory neurons (A and B) or in CHO cells transfected with either DOR or KOR(C). (A) Pretreatment with the 12-/15-LOX-dependent AA metabolites, 12-HETE (100 nM), and 15-HETE (100 nM), alone or in combination, for 15 minutes, prevented inhibition of cAMP accumulation by the KOR agonist, U50488 ( $100 \mathrm{nM})$, but not the 5-HT 1 receptor agonist, 5-CT $(100 \mathrm{nM})$ in BK-pretreated cells. Data represent mean \pm S.E.M. of five separate experiments. ${ }^{*} P<0.05$. (B) In contrast to the effects of 12 - and 15-HETE, pretreatment for 15 minutes with the 5-LOX-dependent AA metabolite 5-HETE (100 nM) had no effect on either DPDPE (DOR) or U50488 (KOR)-mediated inhibition of cAMP accumulation. (C) Pretreatment of transfected CHO cells for 15 minutes with 12-HETE ( $100 \mathrm{nM}$ ) or 15-HETE (100 nM) prevented inhibition of cAMP accumulation by the DOR agonist DPDPE or the KOR agonist U50488 $(100 \mathrm{nM})$, but not the $5-\mathrm{HT}_{1}$ receptor agonist, 5 -CT $(100 \mathrm{nM})$. Data represent mean \pm S.E.M. of four separate experiments. $* P<$ 0.05 versus vehicle pretreatment condition in the absence of opioid agonist. 
5-HETE, eicosapentaenoic acid, leukotriene A4 (LTA4), linolenic acid (LA), and $\alpha$-linolenic acid (ALA) were identified by comparison with synthetic standards using LC-MS/MS. The levels of 12-HETE and 15-HETE were significantly greater in cultures treated with AA compared with vehicle $(* * * P<$ $0.001, n=5$ ). By contrast, treatment with bradykinin did not increase the levels of either 12- or 15-HETE (Fig. 1).

Effect of 12-HETE and 15-HETE on DOR Function Ex Vivo. To determine whether 12- and 15-HETEs altered the responsiveness of the DOR system, we added them to cultures of peripheral sensory neurons where functional competence of the DOR system was induced with BK, as BK pretreatment does not increase 12- or 15-HETE levels (Fig. 1) or produce the refractory, nonresponsive state of the DOR system. As shown in Fig. 2, DPDPE reduced $\mathrm{PGE}_{2}$-stimulated cAMP levels by $40 \% \pm 10 \%$. In cells pretreated with 12 -HETE or 15-HETE alone or in combination, DPDPE did not inhibit $\mathrm{PGE}_{2}$-stimulated cAMP accumulation $(P<0.05, n=5$ experiments). Pretreatment with either 12- or 15-HETE did not alter basal or $\mathrm{PGE}_{2}$-stimulated cAMP accumulation.

To determine whether 12- and 15-HETE reduced responsiveness of other receptor systems expressed by peripheral sensory neurons (Berg et al., 2011), we measured the inhibition of cAMP accumulation by the KOR agonist U50488 (using BK pretreatment to induce functional competence) and the serotonin (5-HT) $)_{1}$ receptor agonist 5-carboxamidotryptamine (5-CT), which does not require pretreatment with an inflammatory mediator to respond to agonist stimulation. As shown in Fig. $3 \mathrm{~A}$, treatment of cells with maximal KOR or $5-\mathrm{HT}_{1}$ agonist concentrations inhibited $\mathrm{PGE}_{2}$-stimulated cAMP levels to a similar degree. Similar to the effects on the response of DOR to DPDPE, pretreatment with 12-HETE and/or 15-HETE also abolished the response to the KOR agonist U50488. By contrast, neither 12-HETE nor 15-HETE, either alone or in combination, altered the response to the $5-\mathrm{HT}_{1}$ receptor agonist, 5-CT. We also tested the effects of the 5-LOX AA metabolite 5-HETE. As shown in Fig. 3B, exogenously applied 5-HETE did not alter the inhibition of adenylyl cyclase activity by either DPDPE or U50488 (Fig. 3B).

In CHO cells heterologously expressing DOR or KOR and that do not require pretreatment with inflammatory mediators to elicit functional competence, pretreatment with 12 - and 15-HETE also prevented DPDPE- and U50488-mediated inhibition of $\mathrm{PGE}_{2}$-stimulated cAMP accumulation (Fig. 3C). By
A

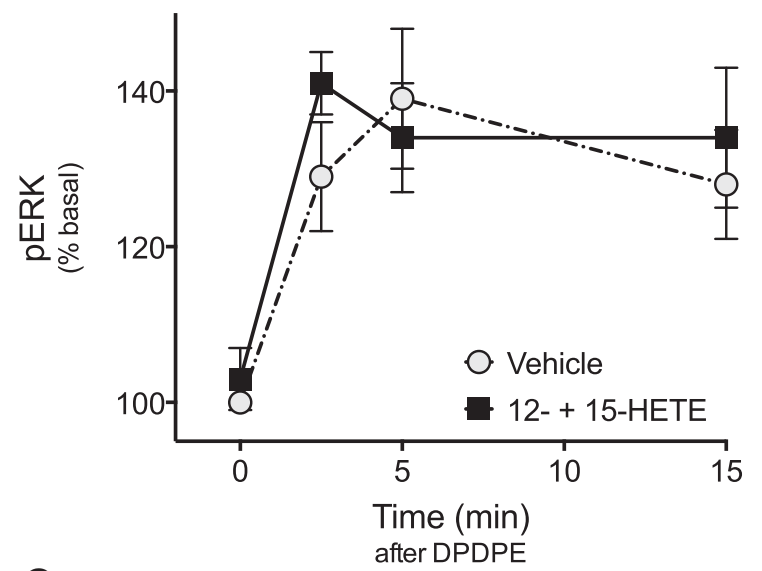

C
$\mathrm{CHO}$

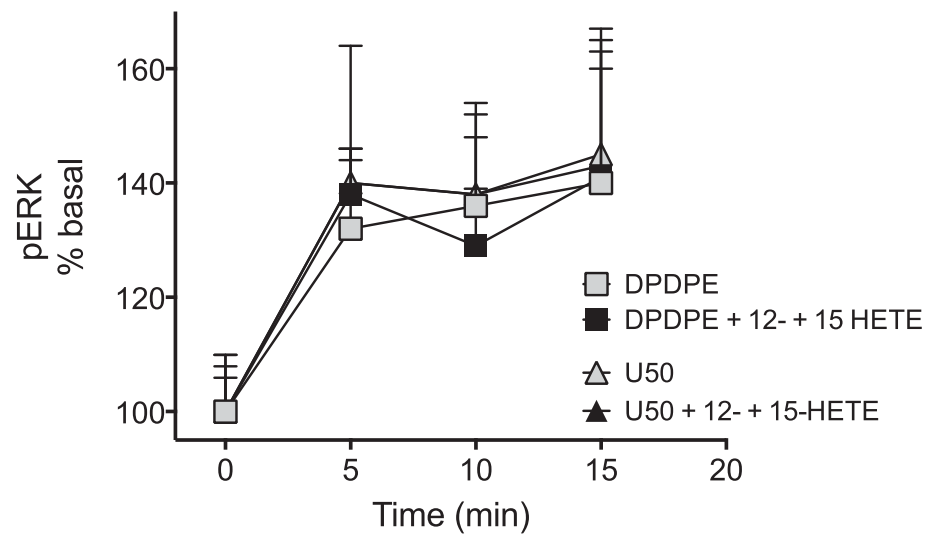

B $\mathrm{KOR}$

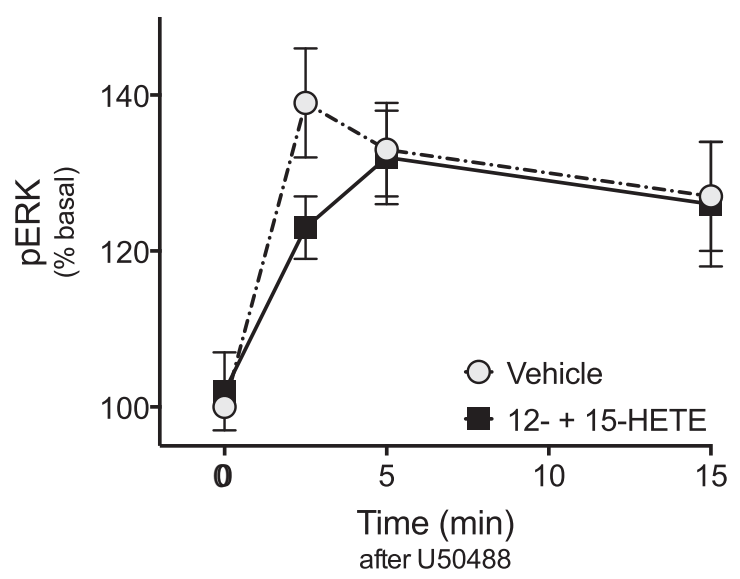

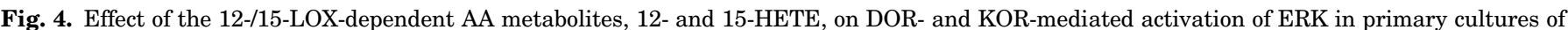

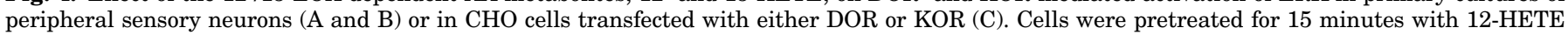

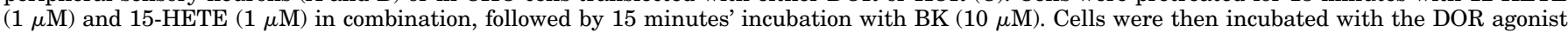

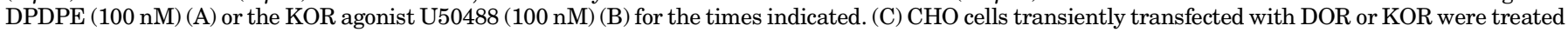

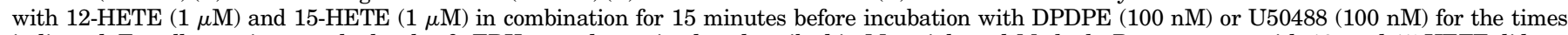

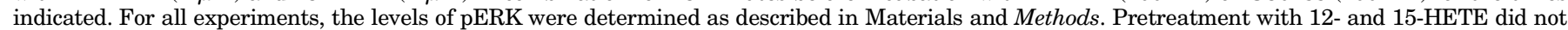

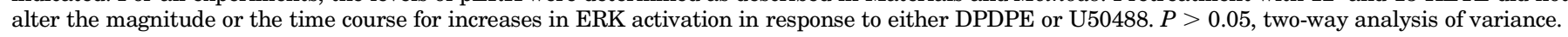


contrast, pretreatment with 12- and 15-HETE had no effect on the ability of endogenously expressed $5-\mathrm{HT}_{1 \mathrm{~B}}$ receptors to inhibit $\mathrm{PGE}_{2}$-stimulated cAMP accumulation.

In addition to inhibition of cAMP signaling, activation of opioid receptors in peripheral nociceptors activates MAPKinase signaling cascades, such as ERK (Berg et al., 2011; Jamshidi et al., 2015). As shown in Fig. 4, the levels of pERK (an index of activation) increased in response to DPDPE (Fig. 4A) and U50488 (Fig. 4B). Administration of a combination of 12-HETE and 15-HETE did not alter the ability of either DPDPE or U50488 to activate ERK in peripheral sensory neurons or in CHO cells (Fig. 4). Taken together, these data suggest that the inhibitory effects of 12- and 15-HETE are both receptor system-dependent (alters opioid but not $5-\mathrm{HT}_{1}$ receptor systems), as well as signaling pathway-selective (alters opioid receptor-mediated inhibition of cAMP signaling but not activation of ERK).

\section{Effect of 12-HETE and 15-HETE on DOR Function In Vivo}

We next tested the effects of exogenous administration of 12- and 15-HETE on DOR responsiveness in a behavioral model of thermal nociception, where DOR functional competence was induced by administration of BK. As shown in Fig. 5, DPDPE reduced completely $\mathrm{PGE}_{2}$-evoked thermal allodynia after injection of $\mathrm{BK}$ into the rat hind paw. Administration of 12-HETE or 15-HETE, either alone or in combination, abolished DPDPE-mediated antiallodynia. Because HETEs have been shown to activate TRPV1 channels, albeit at micromolar concentrations (Hwang et al., 2000), we verified that injection of 12- and 15-HETE alone or in combination did not alter baseline PWL (Supplemental Fig. 1). Supplemental Fig. 2 shows that injection of 12- and 15-HETE did not alter the allodynia produced by intraplantar injection of BK.

\section{Effect of 12/15 LOX Inhibitors on DOR Function Ex Vivo and In Vivo}

Previously, we reported that DOR system functional competence induced by AA treatment is transient, with DOR becoming nonresponsive (i.e., DPDPE becomes ineffective at inhibiting $\mathrm{PGE}_{2}$-stimulated cAMP accumulation) within 15 minutes of washout of AA (Sullivan et al., 2015). Thus, we next determined whether DOR responsiveness after AA treatment would be prolonged by inhibition of LOX. As shown in Fig. 6, pretreatment with a combination of the 12-/15-LOX inhibitors (i.e., LUT and BAIC) (Sadik et al., 2003; Deschamps et al., 2006) prolonged DOR functional competence induced by AA. Consistent with our previous results, the inhibition of cAMP accumulation in response to DPDPE was transient and returned to baseline $\mathrm{PGE}_{2}$-stimulated levels within 15 minutes of washout of AA. By contrast, in cells that were pretreated with the LOX inhibitors, DOR functional competence persisted when tested after 15 minutes of AA washout. Treatment with the LOX inhibitors did not alter either basal or $\mathrm{PGE}_{2}$-stimulated cAMP accumulation.

We have shown previously that after an initial induction of functional competence, intraplantar administration of AA also
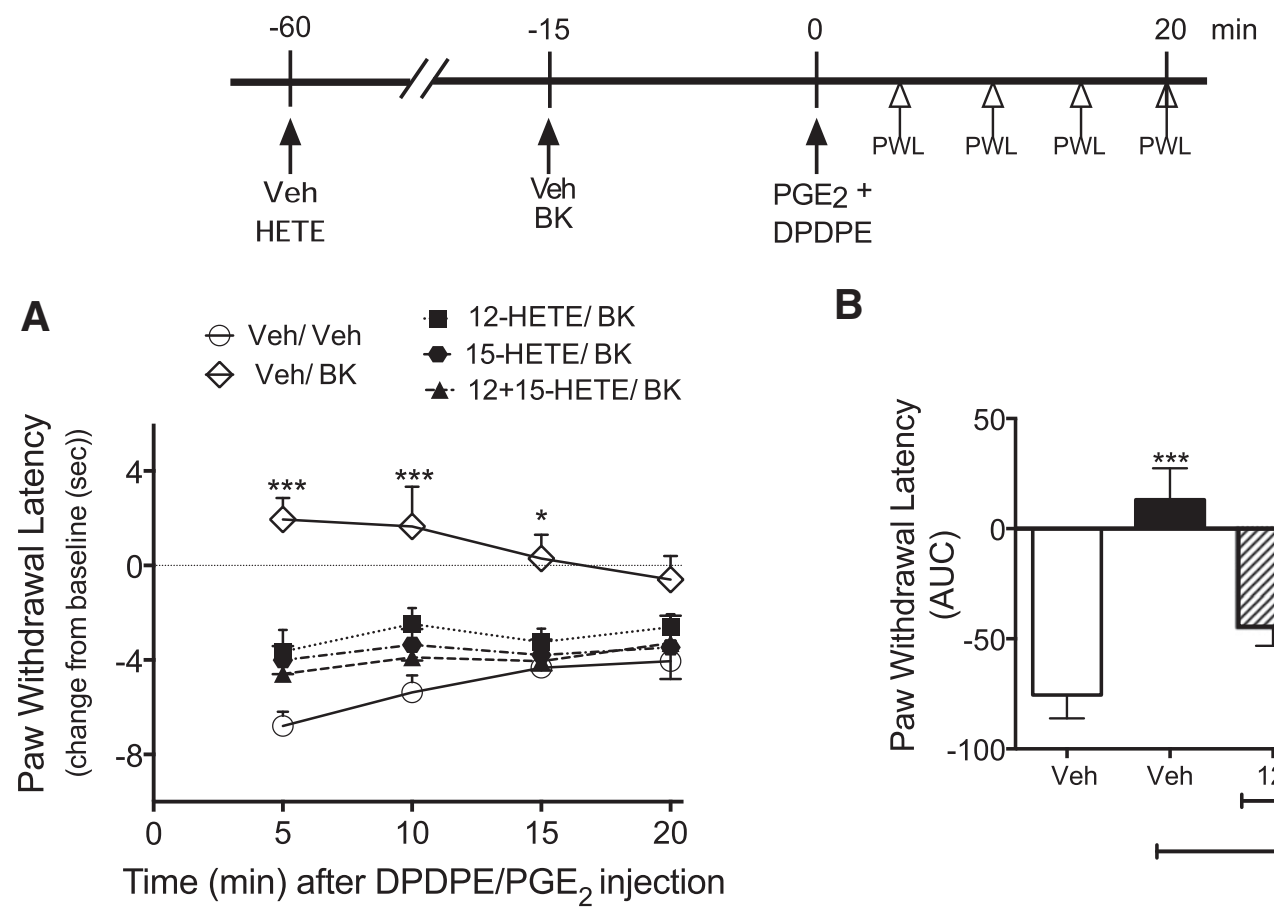

B

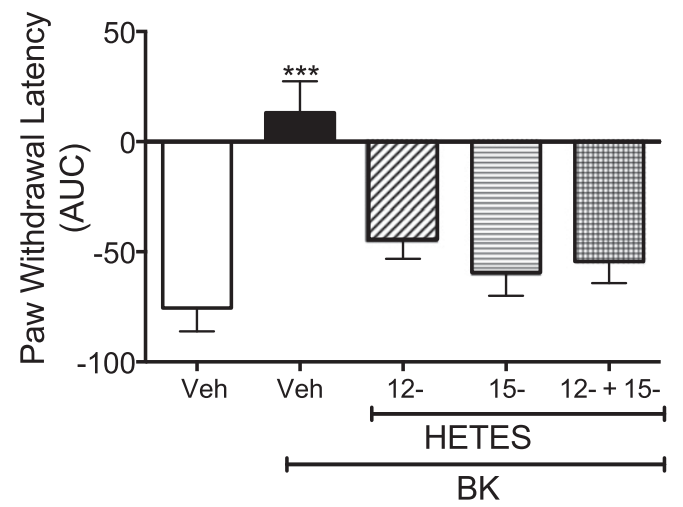

Fig. 5. Pretreatment with the 12-/15-LOX-dependent AA metabolites 12-HETE and 15-HETE attenuates DPDPE-mediated anti-allodynia. Rats received intraplantar injections of vehicle (Veh), 12-HETE $(0.1 \mu \mathrm{g}), 15$-HETE $(0.1 \mu \mathrm{g})$, or 12-HETE and 15-HETE combined. After 45 minutes, rats were injected with Veh or BK $(25 \mu \mathrm{g})$, and then 15 minutes later received coinjections of PGE $_{2}(0.3 \mu \mathrm{g})$ with DPDPE (20 $\left.\mu \mathrm{g}\right)$. (A) PWL was measured before (baseline) and at 5-minute intervals after the $\mathrm{PGE}_{2} / \mathrm{DPDPE}$ injection for 20 minutes. Data were evaluated for statistical differences with two-way analysis of variance (ANOVA), followed by Tukey's post hoc analysis. (B) Area under the individual time-course curves (AUC) for each pretreatment condition. Data were evaluated for statistical differences with one-way ANOVA, followed by Dunnett's post hoc analysis. Data represent mean \pm S.E.M. of 6 (Veh-Veh pretreatment), 9 (Veh-BK pretreatment), 12 (12-HETE/BK pretreatment), 9 (15-HETE/BK pretreatment), or 4 (12- + 15-HETE/BK pretreatment) animals per group. $* P<0.05, * * * P<0.001$ compared with $\mathrm{Veh} / \mathrm{Veh} / \mathrm{DPDPE}+\mathrm{PGE}_{2}$ (i.e., no pretreatment with BK). As shown in Supplemental Fig. 1 and 2, 12-HETE and 15-HETE did not alter either baseline PWL or $\mathrm{PGE}_{2}$-evoked PWL. 

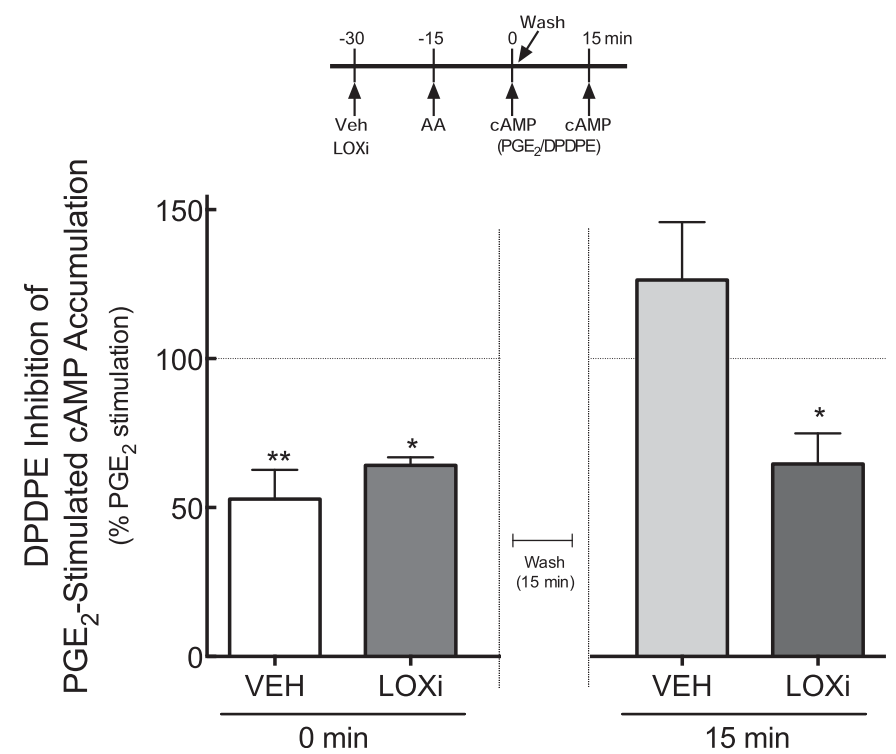

Fig. 6. Inhibition of 12- and 15-LOX activity prolongs DOR functional competence for the inhibition of PGE2-stimulated cAMP accumulation in primary cultures of peripheral sensory neurons. Cells were incubated with or without the combination of 12-/15- LOX inhibitors (LOXi) luteolin $(1 \mu \mathrm{M})$ and BAIC $(1 \mu \mathrm{M})$ for 30 minutes before treatment with $\mathrm{AA}(50 \mu \mathrm{M})$ for 15 minutes. DPDPE (100 nM)-mediated inhibition of $\mathrm{PGE}_{2}$-stimulated cAMP was measured immediately, or cells were washed and DPDPEmediated responses were determined 15 minutes later (see time line). LOX inhibitors did not alter basal or $\mathrm{PGE}_{2}$-stimulated cAMP accumulation, which were $0.6 \pm 0.2 \mathrm{pmol} / \mathrm{well}$ and $205 \% \pm 42 \%$ above basal, respectively. Data represent mean \pm S.E.M. of four experiments $* P<$ 0.05 ; $* * P<0.01$ compared with $\mathrm{PGE}_{2}$ in the absence of DPDPE.

induces a subsequent nonresponsive state of the DOR system that is refractory to reinduction of functional competence by $\mathrm{BK}$, similar to effects that occur in primary cultures of peripheral sensory neurons. Development of this refractory, nonresponsive state is blocked by a nonselective LOX inhibitor (Sullivan et al., 2015). Consistent with our previous data, preinjection of the 12-/15-LOX inhibitors LUT and BAIC restored DPDPE-mediated reduction in $\mathrm{PGE}_{2}$-evoked thermal allodynia following administration of AA (Fig. 7). As shown in Supplemental Fig. 2, injection of the LOX inhibitors did not alter $\mathrm{PGE}_{2}$-evoked thermal allodynia.

DOR-Mediated Reduction of Thermal Nociception is Regulated by 12-LOX and 15-LOX Metabolites in the Carrageenan Model of Inflammatory Pain. Intraplantar injection of carrageenan induces DOR functional competence for reduction of thermal allodynia when measured 15 minutes later, but DOR is nonresponsive when tested 3 hours after injection, even though the allodynia is still present (Sullivan et al., 2015) (Fig. 8, A and B); however, as shown in Fig. 8B, after administration of LOX inhibitors LUT and BAIC, DOR functional competence persisted through 3 hours. These data suggest that, similar to treatment with exogenous AA, carrageenan produces inflammatory mediators that induce functional competence and also produce 12-/15-LOX metabolites (12- and 15-HETE) that promote the nonresponsive state of the DOR system.

\section{Discussion}

Peripheral opioid receptors expressed on pain-sensing neurons are attractive potential targets for development of effective analgesics that would be devoid of severe CNSmediated adverse effects, such as respiratory depression and addiction. As peripherally restricted MOR agonists can produce debilitating constipation (Grunkemeier et al., 2007), attention has shifted to peripheral DOR or KOR. If these receptors are to be valid targets for new drug development, it is important to understand their regulation.

Previous work has shown that peripheral opioid receptor systems expressed by pain-sensing neurons are regulated differently from those in the CNS or expressed heterologously. For example, acting at peripheral opioid receptors, opioid agonists do not promote antinociception or inhibit adenylyl cyclase activity unless cells are first exposed to inflammatory mediators that, acting via a COX-dependent AA metabolite, increase opioid agonist antinociceptive efficacy (Berg et al., 2007a,b, 2011; Sullivan et al., 2015). In addition to increasing opioid agonist efficacy, AA metabolites can also suppress peripheral opioid receptor antinociceptive signaling via the activity of lipoxygenase (LOX) enzymes (Sullivan et al., 2015). Here we identified that the 12/15-LOX-dependent AA metabolites, 12- and 15-HETE, are responsible for the nonresponsive state of the DOR system after administration of AA or carrageenan.

Lipoxygenases (5-, 12- and 15-LOX) are a family of enzymes involved in the oxidative metabolism of AA, and they catalyze the formation of corresponding hydroxyeicosatetraenoic acids (5-, 12- and 15-HETE). Using HPLC tandem mass spectrometry analyses (LC-MS/MS), we found that the 12/15-LOX-AA metabolites, 12-HETE and 15-HETE, but not 5-HETE, increased after treatment of primary cultures of peripheral sensory neurons with exogenously applied AA, but not with BK. Administration of 12-HETE and 15-HETE, either alone or in combination, blocked completely the inhibition of $\mathrm{PGE}_{2^{-}}$ stimulated cAMP accumulation in response to the DOR agonist DPDPE, whereas administration of 5-HETE had no effect. In vivo, intraplantar injection of either 12- or 15-HETE blocked completely DOR-mediated inhibition of $\mathrm{PGE}_{2}$-evoked thermal allodynia. Selective inhibitors of 12- and 15-LOX prevented the reduced responsiveness of the DOR system after AA treatment ex vivo and after AA and carrageenan treatment in vivo. These results suggest that 12 - and 15-HETEs derived from the metabolism of AA by LOX are responsible for the refractory, nonresponsiveness state of the DOR system in peripheral sensory neurons.

The observation that BK administration was not effective at reinducing DOR responsiveness after exogenous administration of AA or after carrageenan injection suggests that perhaps 12- and 15-HETEs could interfere with BK signaling systems and the induction of opioid receptor system functional competence in peripheral sensory neurons; however, the allodynia produced by intraplantar injection of BK into the hind paw was not altered by exogenous AA (Sullivan) or by 12-/15-HETEs. Further, both 12- and 15-HETE blocked the inhibition of $\mathrm{PGE}_{2}$-stimulated cAMP accumulation in CHO cells heterologously expressing DOR or KOR, which do not require pretreatment with $\mathrm{BK}$ to induce responsiveness as do peripheral sensory neurons. These data suggest that the $12 / 15$ LOX metabolites interfere with signaling by DOR and KOR.

When applied at micromolar concentrations, HETEs have been shown to activate transient receptor potential vanilloid-1 (TRPV1) channels and produce thermal hyperalgesia (Hwang 


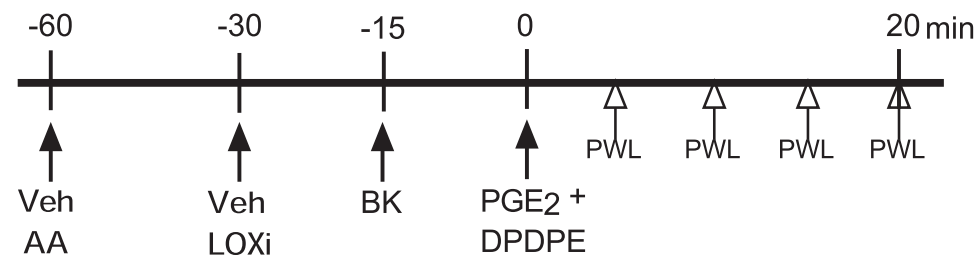

\section{$\diamond$ Veh / Veh / BK • AA / Veh / BK $\ominus \mathrm{AA} / \mathrm{LOXi} / \mathrm{BK}$}

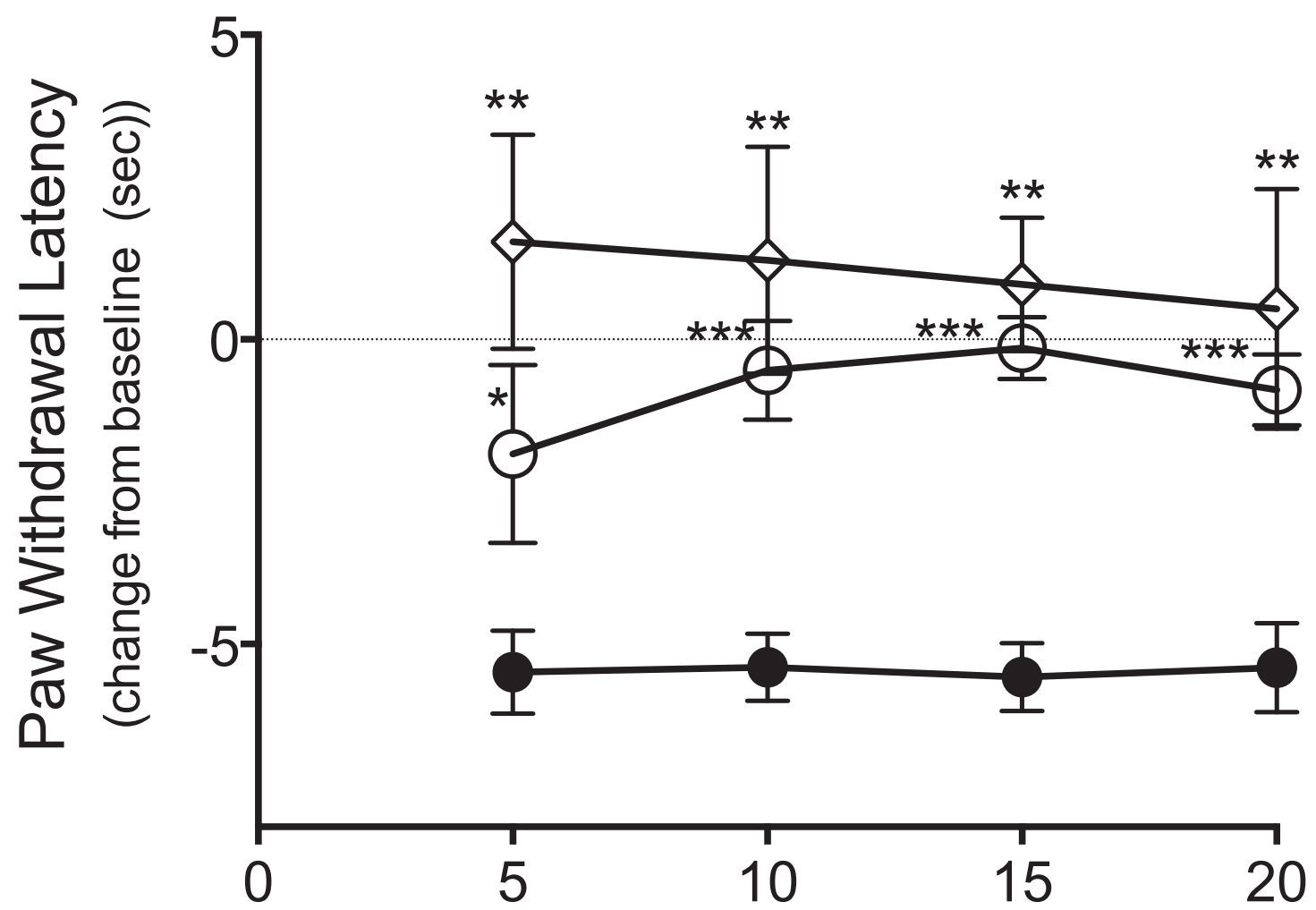

Time after DPDPE/PGE 2 injection (min)

Fig. 7. Inhibition of 12- and 15-LOX activity restores DOR system responsiveness for the reduction of $\mathrm{PGE}_{2}$-evoked thermal allodynia after AA treatment. Rats received injections of AA ( $3 \mu \mathrm{g}, 50 \mu \mathrm{l}$, intraplantarly) 30 minutes before injections of Veh or the LOX inhibitors (LOXi) LUT ( $3 \mu \mathrm{g})$ and BAIC $(3 \mu \mathrm{g})$ in combination (50 $\mu \mathrm{l}$, intraplantarly). Rats were then injected with BK (25 $\mu \mathrm{g}$, intraplantarly) 15 minutes later to induce functional competence; 15 minutes after BK administration, DOR responsiveness was assessed by injecting PGE $\mathrm{P}_{2}(0.3 \mu \mathrm{g})$ along with DPDPE $(20 \mu \mathrm{g})(50 \mu \mathrm{l}$, intraplantarly) and PWLs were measured before (baseline) and at 5-minute intervals after injection for 20 minutes. Data represent mean \pm S.E.M. of the change from baseline PWL, six animals per group. ${ }^{*} P<0.05$; $* * P<0.01$; $* * * P<0.001$ compared Veh-Veh.

et al., 2000; Sisignano et al., 2013; Kelly et al., 2015). Thus, it is possible that the reduced antinociceptive signaling by peripherally restricted DOR agonists is due to functional antagonism produced by 12- or 15-HETEs activating TRPV1; however, baseline PWL to thermal stimulation of the hind paw was not altered by intraplantar injection of 12- or 15-HETE at a dose of $0.1 \mu \mathrm{g}$ (calculated to produce a concentration of $\approx 300 \mathrm{nM}$, assuming a volume of distribution of $1 \mathrm{ml}$ in the paw), indicating that hyperalgesia produced by activation of TRPV1 channels by the HETEs was not responsible for the reduced antinociceptive effect of DOR agonists in vivo after AA or carrageenan.

Although it has been reported that 12-LOX-dependent AA metabolites mediate presynaptic inhibition of neurotransmitter release in neurons of the periaqueductal gray (Vaughan et al., 1997) and nucleus raphe magnus (Zhang and Pan, 2012) in response to opioid receptor activation, there are no reports of regulation of opioid receptor function by LOX metabolites. Since the 12- and 15-HETES reduced DOR and KOR receptor signaling, but not signaling of the $5-\mathrm{HT}_{1}$ receptor, it is unlikely 
A

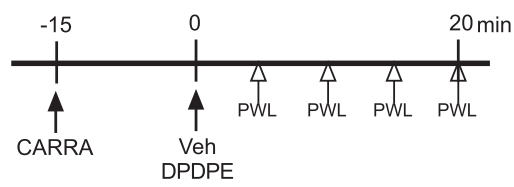

DPDPE

- Veh

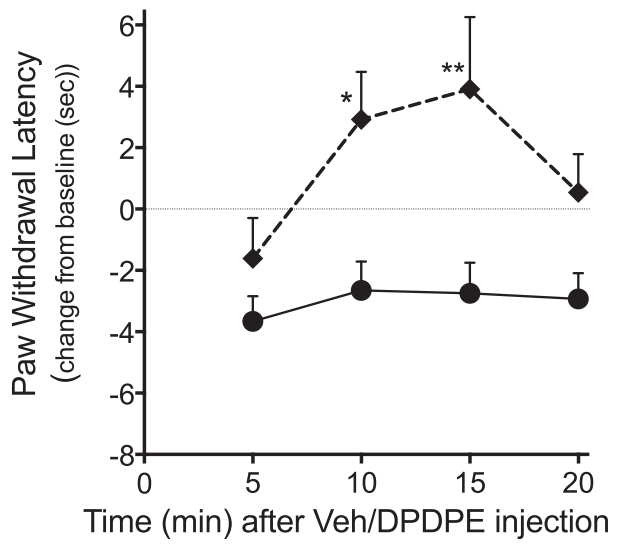

B

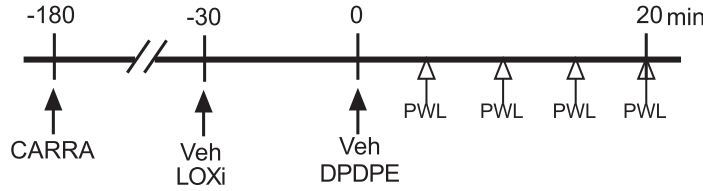

CARRA / Veh / Veh

CARRA / Veh / DPDPE

$\ominus$ CARRA / LOXi / Veh

$\Leftrightarrow$ CARRA / LOXi / DPDPE

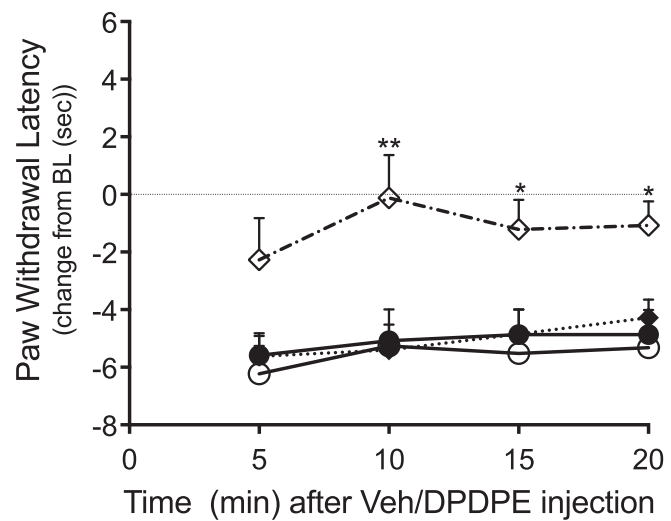

Fig. 8. The effect of LOX inhibitors on the duration of DOR system responsiveness to DPDPE on carrageenan-induced thermal allodynia. (A) Then, 15 minutes after intraplantar injection of carrageenan (CARRA, $500 \mu \mathrm{g}$ ), thermal allodynia was reduced completely by DPDPE (20 $\mu \mathrm{g})$ Data represent mean \pm S.E.M. of six rats/group. ${ }^{*} P<0.05 ; * * P<0.01$ from CARRA-Veh. (B) Three hours after carrageenan injection, DPDPE no longer reduced thermal allodynia. After injection of the 12-/15-LOX inhibitors, reduction of carrageenan-induced thermal allodynia by DPDPE was restored. As shown in the time line, rats received intraplantar injections of Veh or a combination of the 12-LOX inhibitor, LUT ( $3 \mu \mathrm{g})$, and the 15-LOX inhibitor BAIC ( $3 \mu \mathrm{g})$ 2.5 hours after injection of carrageenan $(500 \mu \mathrm{g})$. DPDPE $(20 \mu \mathrm{g})$ was administered 30 minutes after LOX inhibitor pretreatment (i.e., responses to DPDPE were determined 3 hours after carrageenan administration). Data represent mean \pm S.E.M. of six rats/group. ${ }^{*} P<0.05 ; * * P<0.01$ from CARRA-Veh-DPDPE.

that the HETES target postreceptor mechanisms to reduce opioid receptor function in peripheral sensory neurons. 12-HETE has been shown to interact directly with G protein-coupled receptors, acting as an antagonist at thromboxane receptors (Siangjong et al., 2013) and as an agonist at GPR31 receptors (Powell and Rokach, 2015). It is unlikely that 12- or 15-HETE acts as an antagonist at DOR or KOR orthosteric binding sites because ERK activation in response to either DPDPE or U50488 was not altered in the presence of the HETEs. Our current working hypothesis is that 12- and 15-HETE may be allosteric regulators of DOR and KOR to reduce signaling through antinociceptive pathways $(\mathrm{Gi})$, but not ERK.

In contrast to the inhibitory effects on DOR function in response to exogenous administration of AA and the inflammatory conditions induced by carrageenan, it is curious that administration of the inflammatory mediator BK did not promote a refractory nonresponsive state of the DOR system (Sullivan et al., 2015). BK treatment leads to endogenous production of AA that is metabolized by COX to induce functional competence of opioid receptors in peripheral sensory neurons (Patwardhan et al., 2005; Berg et al., 2007a). It is possible that BK activation of bradykinin $\mathrm{B} 2$ receptors and Gq-protein-mediated phospholipid signaling elicits compartmentalized release of AA that is available for metabolism by COX, but not by 12-/15-LOX enzymes in nociceptors. Preferential metabolism of AA by certain enzymes in cells has been reported. For example, activation of cytosolic phospholipase $\mathrm{A}_{2}$ signaling by $5-\mathrm{HT}_{2 \mathrm{~A}}$ and $5-\mathrm{HT}_{2 \mathrm{C}}$ receptors results in differential AA metabolism by cytochrome $\mathrm{P} 450$ versus COX enzymes, respectively (Berg et al., 1998). Further, activation of protease-activated receptors 1 and 4, which stimulate cytosolic phospholipase A2 signaling in platelets, differentially elicits AA metabolism by COX-1 versus 12 -LOX, respectively (Holinstat et al., 2011).

After initial induction of DOR system responsiveness by intraplantar injection of carrageenan, the DOR system became unresponsive to agonist activation when tested 3 hours after carrageenan. Responsiveness was restored by inhibitors of 12-/15-LOX. Carrageenan elicits an endogenous inflammatory response that mimics that which occurs naturally, suggesting that the nonresponsive state of the peripheral DOR system could also occur in inflammatory conditions where 12-/15-LOX metabolites of AA are generated, such as rheumatoid arthritis (Deleuran et al., 1994; Liagre et al., 1997) and osteoarthritis (Kelly et al., 2015). It is possible that a LOX-dependent nonresponsive state of peripheral opioid receptor systems may underlie the cases where some clinical studies have failed to demonstrate effectiveness of peripherally restricted opioids for the treatment of pain (for reviews, see Vadivelu et al., 2011; Nielsen et al., 2015).

In summary, we have identified 12- and 15-HETE as the LOX-dependent AA metabolites that produce a refractory, nonresponsiveness state of the DOR system for antinociceptive signaling in peripheral pain-sensing neurons that occurs after exogenous administration of AA or carrageenan. Inhibition of 12/15 LOX extended the duration of responsiveness of the DOR system to at least 3 hours. Since 12/15 LOX metabolites are produced in a variety of inflammatory states, inhibition of $12 / 15$ LOX activities may be an important adjunct for sustained peripheral opioid receptor function that will allow peripherally restricted opioid medications to block pain neurotransmission 
to the CNS without the dose-limiting and life-threatening CNS-mediated adverse effects.

\section{Acknowledgments}

The authors thank Dr. Bill Clarke for helpful discussions and comments.

\section{Authorship Contributions}

Participated in research design: Berg, Sullivan.

Conducted experiments: Chavera, Pando, Gao, Sullivan.

Performed data analysis: Berg, Gao, Sullivan.

Wrote or contributed to the writing of the manuscript: Berg, Sullivan.

\section{References}

Berg KA, Maayani S, and Clarke WP (1998) Interactions between effectors linked to serotonin receptors. Ann N Y Acad Sci 861:111-120.

Berg KA, Patwardhan AM, Sanchez TA, Silva YM, Hargreaves KM, and Clarke WP (2007a) Rapid modulation of micro-opioid receptor signaling in primary sensory neurons. J Pharmacol Exp Ther 321:839-847.

Berg KA, Rowan MP, Gupta A, Sanchez TA, Silva M, Gomes I, McGuire BA, Portoghese PS, Hargreaves KM, Devi LA, et al. (2012) Allosteric interactions between $\delta$ and $\kappa$ opioid receptors in peripheral sensory neurons. Mol Pharmacol 81:264-272.

Berg KA, Rowan MP, Sanchez TA, Silva M, Patwardhan AM, Milam SB, Hargreaves KM, and Clarke WP (2011) Regulation of $\kappa$-opioid receptor signaling in peripheral sensory neurons in vitro and in vivo. J Pharmacol Exp Ther 338:92-99.

Berg KA, Zardeneta G, Hargreaves KM, Clarke WP, and Milam SB (2007b) Integrins regulate opioid receptor signaling in trigeminal ganglion neurons. Neuroscience 144:889-897.

Deleuran B, Iversen L, Kristensen M, Field M, Kragballe K, Thestrup-Pedersen K, and Stengaard-Pedersen K (1994) Interleukin-8 secretion and 15-lipoxygenase activity in rheumatoid arthritis: in vitro anti-inflammatory effects by interleukin-4 and interleukin-10, but not by interleukin-1 receptor antagonist protein. $\mathrm{Br} J$ Rheumatol 33:520-525.

Deschamps JD, Kenyon VA, and Holman TR (2006) Baicalein is a potent in vitro inhibitor against both reticulocyte 15-human and platelet 12-human lipoxygenases. Bioorg Med Chem 14:4295-4301.

Gao X, Zhang Q, Meng D, Isaac G, Zhao R, Fillmore TL, Chu RK, Zhou J, Tang K, Hu Z et al. (2012) A reversed-phase capillary ultra-performance liquid chromatographymass spectrometry (UPLC-MS) method for comprehensive top-down/bottom-up lipid profiling. Anal Bioanal Chem 402:2923-2933.

Gendron L, Mittal N, Beaudry H, and Walwyn W (2015) Recent advances on the $\delta$ opioid receptor: from trafficking to function. $\mathrm{Br} J$ Pharmacol 172:403-419.

Grunkemeier DM, Cassara JE, Dalton CB, and Drossman DA (2007) The narcotic bowel syndrome: clinical features, pathophysiology, and management. Clin Gastroenterol Hepatol 5:1126-1139, quiz 1121-1122.

Hargreaves K, Dubner R, Brown F, Flores C, and Joris J (1988) A new and sensitive method for measuring thermal nociception in cutaneous hyperalgesia. Pain 32 $77-88$.

Holinstat M, Boutaud O, Apopa PL, Vesci J, Bala M, Oates JA, and Hamm HE (2011) Protease-activated receptor signaling in platelets activates cytosolic phospholipase A2 $\alpha$ differently for cyclooxygenase-1 and 12-lipoxygenase catalysis. Arterioscler Thromb Vasc Biol 31:435-442.
Hwang SW, Cho H, Kwak J, Lee SY, Kang CJ, Jung J, Cho S, Min KH, Suh YG, Kim D, et al. (2000) Direct activation of capsaicin receptors by products of lipoxygenases: endogenous capsaicin-like substances. Proc Natl Acad Sci USA 97: $6155-6160$

Jamshidi RJ, Jacobs BA, Sullivan LC, Chavera TA, Saylor RM, Prisinzano TE, Clarke WP, and Berg KA (2015) Functional selectivity of kappa opioid receptor agonists in peripheral sensory neurons. J Pharmacol Exp Ther 355:174-182.

Kelly S, Chapman RJ, Woodhams S, Sagar DR, Turner J, Burston JJ, Bullock C, Paton K, Huang J, Wong A, et al. (2015) Increased function of pronociceptive TRPV1 at the level of the joint in a rat model of osteoarthritis pain. Ann Rheum Dis 74:252-259.

Lee JH and Kim GH (2010) Evaluation of antioxidant and inhibitory activities for different subclasses flavonoids on enzymes for rheumatoid arthritis. J Food Sci 75: $\mathrm{H} 212-\mathrm{H} 217$.

Liagre B, Vergne P, Rigaud M, and Beneytout JL (1997) Expression of arachidonate platelet-type 12-lipoxygenase in human rheumatoid arthritis type B synoviocytes. FEBS Lett 414:159-164.

Nielsen BN, Henneberg SW, Schmiegelow K, Friis SM, and Rømsing J (2015) Peripherally applied opioids for postoperative pain: evidence of an analgesic effect? A systematic review and meta-analysis. Acta Anaesthesiol Scand 59:830-845.

Patwardhan AM, Berg KA, Akopain AN, Jeske NA, Gamper N, Clarke WP, and Hargreaves KM (2005) Bradykinin-induced functional competence and trafficking of the delta-opioid receptor in trigeminal nociceptors. $J$ Neurosci 25 8825-8832.

Patwardhan AM, Diogenes A, Berg KA, Fehrenbacher JC, Clarke WP, Akopian AN, and Hargreaves KM (2006) PAR-2 agonists activate trigeminal nociceptors and induce functional competence in the delta opioid receptor. Pain 125:114-124.

Powell WS and Rokach J (2015) Biosynthesis, biological effects, and receptors of hydroxyeicosatetraenoic acids (HETEs) and oxoeicosatetraenoic acids (oxo-ETEs) derived from arachidonic acid. Biochim Biophys Acta 1851:340-355.

Rowan MP, Ruparel NB, Patwardhan AM, Berg KA, Clarke WP, and Hargreaves KM (2009) Peripheral delta opioid receptors require priming for functional competence in vivo. Eur $J$ Pharmacol 602:283-287.

Sadik CD, Sies H, and Schewe T (2003) Inhibition of 15-lipoxygenases by flavonoids: structure-activity relations and mode of action. Biochem Pharmacol 65:773-781.

Siangjong L, Gauthier KM, Pfister SL, Smyth EM, and Campbell WB (2013) Endothelial 12(S)-HETE vasorelaxation is mediated by thromboxane receptor inhibition in mouse mesenteric arteries. Am J Physiol Heart Circ Physiol 304:H382-H392.

Sisignano M, Angioni C, Ferreiros N, Schuh CD, Suo J, Schreiber Y, Dawes JM, Antunes-Martins A, Bennett DL, McMahon SB, et al. (2013) Synthesis of lipid mediators during UVB-induced inflammatory hyperalgesia in rats and mice. PLoS One 8:e81228.

Stein C (2016) Opioid receptors. Annu Rev Med 67:433-451.

Stein C and Lang LJ (2009) Peripheral mechanisms of opioid analgesia. Curr Opin Pharmacol 9:3-8.

Sullivan LC, Berg KA, and Clarke WP (2015) Dual regulation of $\delta$-opioid receptor function by arachidonic acid metabolites in rat peripheral sensory neurons. $J$ Pharmacol Exp Ther 353:44-51.

Vadivelu N, Mitra S, and Hines RL (2011) Peripheral opioid receptor agonists for analgesia: a comprehensive review. J Opioid Manag 7:55-68.

van Leyen K, Kim HY, Lee SR, Jin G, Arai K, and Lo EH (2006) Baicalein and 12/15lipoxygenase in the ischemic brain. Stroke 37:3014-3018.

Address correspondence to: Dr. Kelly A. Berg, Department of Pharmacology, MS 7764, University of Texas Health Science Center at San Antonio, 7703 Floyd Curl Dr., San Antonio, TX 78229-3900. E-mail: berg@uthscsa.edu 